\title{
Prediction of Intraoperative Fracture by Hammering Sound Frequency Analysis and Stress Estimation during Total Hip Arthroplasty
}

\author{
Rina Sakai $^{1}$ (D) Takeaki Yamamoto ${ }^{2}$, Katsufumi Uchiyama ${ }^{3}$, Kensuke Fukushima3 $^{3}$, \\ Naonobu Takahira ${ }^{1,3}$, Kazuhiro Yoshida ${ }^{1}$, Masanobu Ujihira ${ }^{1}$ \\ ${ }^{1}$ Department of Biomedical Engineering and Technology, Kitasato University School of Allied Health Sciences, \\ Kanagawa, Japan; ${ }^{2}$ Department of Orthopedic Surgery, St. Marianna University School of Medicine, Kanagawa, \\ Japan; ${ }^{3}$ Department of Orthopedic Surgery, Kitasato University School of Medicine, Kanagawa, Japan
}

Correspondence to: Rina Sakai, rinax@kitasato-u.ac.jp

Keywords: Periprosthetic Fracture, Total Hip Arthroplasty, Hammering Sound, Frequency Analysis Received: April 24, $2020 \quad$ Accepted: June 20, 2020 Published: June 23, 2020

Copyright $\odot 2020$ by author(s) and Scientific Research Publishing Inc.

This work is licensed under the Creative Commons Attribution International License (CC BY 4.0).

http://creativecommons.org/licenses/by/4.0/

\section{(c) (i) Open Access}

\section{ABSTRACT}

When a stem is inserted into the femur during total hip arthroplasty, sufficient fixation depends on the surgeon's experience. An objective method of evaluating whether the stem has been correctly fixed may aid clinicians in their decision. We examined the relationship between the sound frequency caused by hammering the stem and the internal stress in artificial femurs, and evaluated the utility of sound frequency analysis to prevent intraoperative fracture. Surgeons inserted one of two types of cementless stems (SL-PLUS and modified CLS) using routine operational procedures into 13 artificial femurs. These are the standard Zweymüllers used in Europe. The difference is the lateral shape; SL-PLUS has holes for removal and the modified CLS has fins to prevent rotation. We estimated stress in the femur via finite element analysis, measured the hammering force, and recorded the sound of hammering for frequency analysis. Finite element analysis revealed that the hammering sound frequency decreased as the maximum stress increased. A decrease in frequency suggested that fixation was sufficient and that continued hammering would increase the risk of fracture. Thus, evaluation of the change in sound frequency during stem insertion may indicate when the hammering force should be reduced, thereby preventing intraoperative periprosthetic fractures. Further frequency change may also predict fractures prior to visual confirmation. We concluded that sound frequency analysis has potential as an objective evaluation method to help prevent intraoperative periprosthetic fractures during stem insertion. 


\section{INTRODUCTION}

Cementless fixation for total hip arthroplasty has been increasingly performed in recent years. Time-saving techniques are particularly important for the elderly and patients with cardiopulmonary complications [1]. However, Moroni et al. reported intraoperative femur fractures in $4.1 \%$ to $27.8 \%$ of patients undergoing cementless fixation $[2,3]$, which can delay the start of postoperative rehabilitation and inhibit patients' ability to resume normal daily activities $[4,5]$.

In cementless fixation, surgeons believe intense hammering is necessary to achieve a strong fix between the stem and femur. At present, the assessment of fixation state depends on the experience and judgment of the surgeon, and intraoperative fractures are unavoidable. Experience suggests that the hammering sound changes as the stem is inserted, and the relative positions of the stem and femur change. Pastrav et al. (2009) and Sakai et al. (2011) suggested that frequency analysis of the sound generated by hammering the stem can be an objective evaluation of stem fixation $[6,7]$.

As no special equipment for sound frequency analysis during stem insertion has been developed, we used commercially available equipment in the present and previous studies. We previously compared the hammering sound frequency and maximum stress in artificial femurs [7, 8]. In this study, we investigated the relationship between sound frequency and stress as a stem insert was hammered into an artificial femur, and identified changes that may occur just before periprosthetic femur fracture.

\section{METHODS}

\subsection{Materials}

Of the fifteen artificial femurs (Sawbones, USA, WA) initially examined, those that were fractured during medullary cavity formation or where stem insertion had caused sinking were excluded, leaving thirteen for sound frequency analysis. Two Zweymüller-type cementless stems differing in shape were used: the SL-PLUS (Smith \& Nephew KK, Japan) and the modified CLS (AI-HIP, Aimedic, Italy). The stem size was determined by specialist hip orthopedists after implant templating from X-ray films of the test femurs. Size 12 was considered suitable, but the size was fixed ideally to prevent bone fracture. Therefore, stems that were three sizes larger were selected.

\subsection{Hammering Sound and Force}

Each artificial femur was fixed in a vice mounted on an experimental table (Figure 1(a)). A digital camera (Lumix DMC-FZ50, Panasonic, Japan) was placed to the side with a load sensor (LMR-S-20KN SA2-P, Kyowa, Japan) attached to the stem inserter (Figure 1(b)), and a microphone (F710, Sony, Japan) was placed $1 \mathrm{~m}$ from the contact plane between the stem inserter and hammer. The femoral cavity was prepared by routine procedures, and the stem was inserted by an orthopedist with more than ten years of experience. Although the number of hammer impacts for stem insertion was not set, hammering continued until the femur fractured. After each impact, the posterior surface of the femur was photographed using the digital camera (Lumix DMC-FZ50, Panasonic, Japan).

The hammering force was defined as the force on the contact plane between the stem inserter and the hammer. Output from the force sensor was recorded by a measurement control computer (LaVieLJ700/E, NEC, Japan) using a dynamic strain meter (DPM-603A, Kyowa, Japan) and a digital storage oscilloscope (DSO2250, Labros, Japan). From data input into the computer, power spectra of hammering sounds were obtained using a Fourier analysis software package (Nov@TEK v.6.10.1, Labros, Japan) and changes in the peak frequency of the maximum amplitude were clarified.

\subsection{Finite Element Analysis}

A finite element model composed of the femur, stem, and stem inserter was constructed using LS-Prepost 2.4 (Livemore Software Technology Corporation, CA, USA) with hexahedral isoparametric 
elements (Figure 2) $[9,10]$. The material properties of the femur, stem and inserter used in the model are shown in Table $1[11,12]$.

(a)

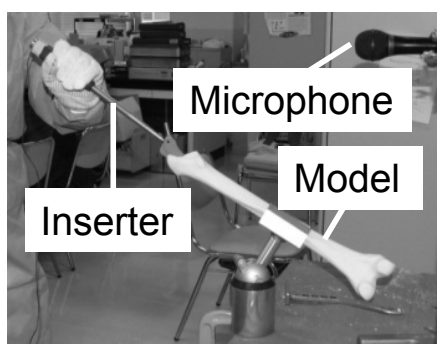

(b)

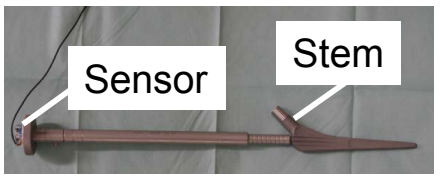

Figure 1. Experimental arrangement. Hammering of a hip stem through an inserter. (a) Attachment of the artificial femur and position of the microphone. (b) Position of the load sensor.

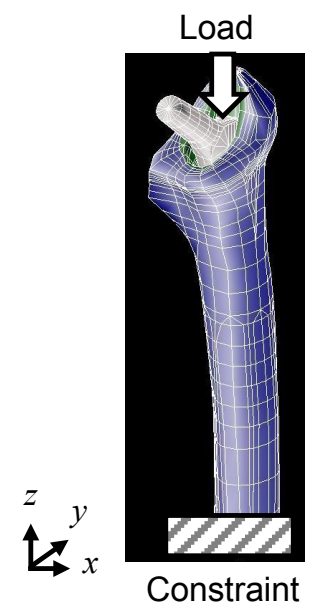

Figure 2. Computational model showing three-dimensional finite elements. The stem was implanted in the femur. The arrow indicates the load direction. The distal end of the femur was constrained in all directions.

Table 1. Properties of materials used in the finite element model.

\begin{tabular}{ccccc}
\hline & \multicolumn{3}{c}{ Properties } \\
\cline { 2 - 5 } Material & $\begin{array}{c}\text { Coefficient of } \\
\text { elasticity } \\
(\mathrm{GPa})\end{array}$ & $\begin{array}{c}\text { Yield stress } \\
(\mathrm{GPa})\end{array}$ & Poisson's ratio & $\begin{array}{c}\text { Mass density } \\
\left(\mathrm{g} / \mathrm{cm}^{3}\right)\end{array}$ \\
\hline Femur & 10.0 & 0.17 & 0.37 & 2.3 \\
Stem & 105 & 0.95 & 0.30 & 4.5 \\
Inserter & 20,000 & 20 & 0.30 & 290 \\
\hline
\end{tabular}


Dynamic explicit finite element analysis was performed using LS-DYNA ver. 971 (Terrabyte, Japan) software running on an Endeavor Pro-4500 (Epson, Japan) desktop computer. The measured hammering force was used as a loading condition and internal stress in the femoral model was estimated using the following equation:

$$
f=\sigma_{x, y, z} A
$$

where $f$ is the measured hammering force, $\sigma$ is internal stress, and $A$ is the contact area of the medullary cavity and stems. This model used the measured hammering force as a loading condition, and estimated the internal stress in the femur and the maximum stress generated by each hammer strike.

\section{RESULTS}

The high stress areas were distributed in a wide area on the femoral diaphysis following SL-PLUS insertion (Figure 3(a)). The high stress area was distributed in the proximal femur following the modified CLS insertion (Figure 3(b)).

With the SL-PLUS, the peak frequency of the hammering sound decreased to $2.2 \mathrm{kHz}$ compared with the value of $4.4 \mathrm{kHz}$ immediately after stem insertion, and the frequency slightly increased thereafter. Immediately after the decrease in peak frequency, the Von Mises stress inside the femur estimated by finite element analysis increased (Figure 4) and exceeded the yield stress of cortical bone.

With the modified CLS, the peak frequency of the hammering sound decreased to $2.0 \mathrm{kHz}$ compared with the value of $4.4 \mathrm{kHz}$ immediately after stem insertion. After the decrease in the peak frequency, the Von Mises stress inside the femur obtained by finite element analysis exceeded $170 \mathrm{MPa}$ [11], which is the yield stress of cortical bone (Figure 5).

The characteristics of the change in sound frequency according to the number of hammer strikes differed depending on which stem was used; with both stem designs, the peak frequency decreased initially, but the internal stress of the femur exceeded the yield stress of cortical bone before the confirmation of fracture.

(a)

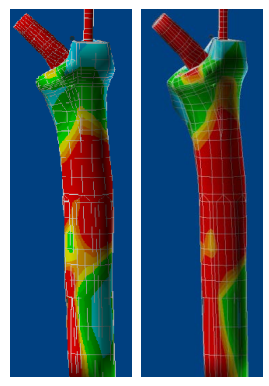

(b)

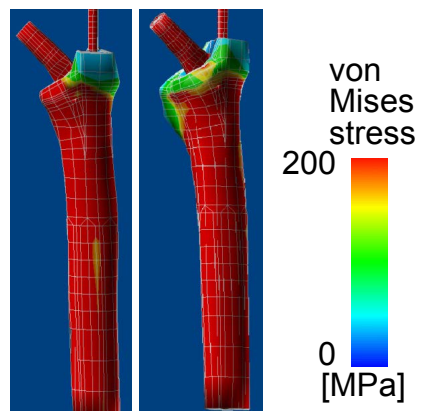

Figure 3. The von Mises stress distribution at the time of fracture determined by the simulation. The red area indicates regions with high von Mises stress. (a) SL-PLUS stem. (b) Modified CLS stem. 


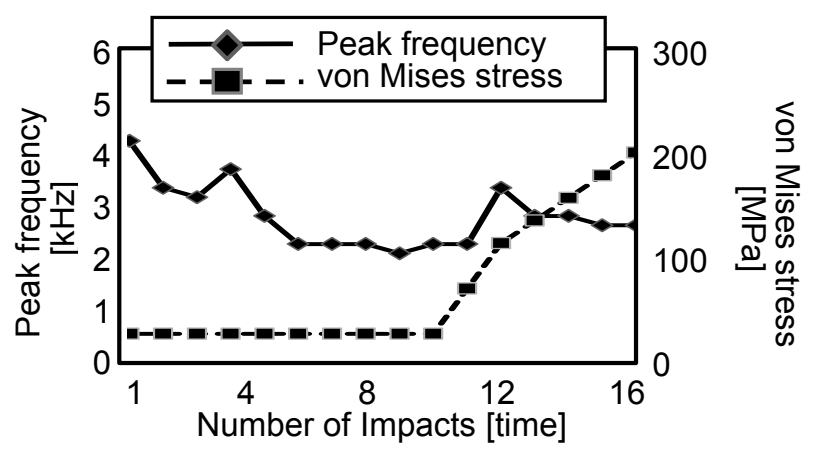

Figure 4. Changes in the von Mises stress and the peak frequency when an SL-PLUS stem was inserted into a femur.

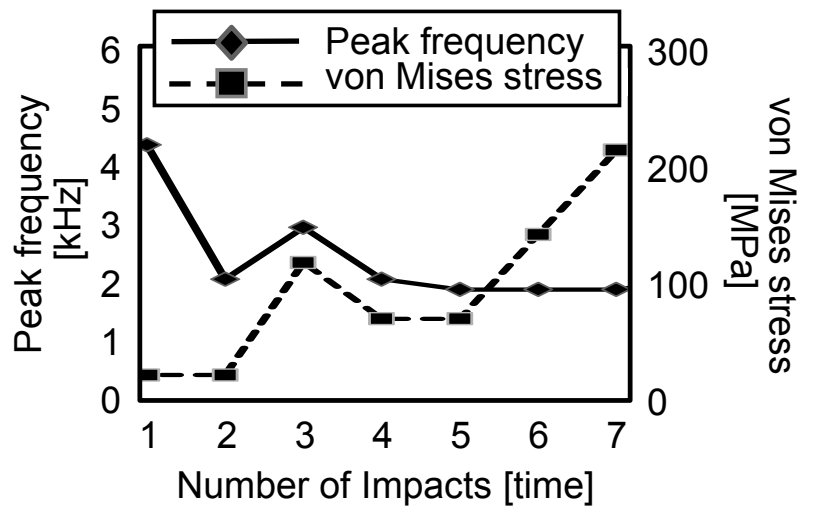

Figure 5. Changes in the von Mises stress and peak frequency when a modified CLS stem was inserted into a femur.

\section{DISCUSSION}

The change in hammering sound is due to the natural frequency of an object being inversely proportional to its length, and the length decreases as the stem is inserted. Before fixation, the peak frequency is only affected by the length of the stem, but as insertion progresses, the sound frequency is affected by the natural frequencies of both the stem and the femur.

Therefore, the decrease in peak frequency may reflect sufficient fixation of the stem to the femur. For both insert designs, a decrease in peak frequency of approximately $3000 \mathrm{~Hz}$ suggested that fixation was sufficient; further hammering and insertion may increase the risk of bone fracture. Thus, periprosthetic fracture may be prevented by stopping the impact when the peak frequency begins to decrease.

In both cases, the finite element analysis revealed that the stress at the fracture site exceeded $170 \mathrm{MPa}$, which is the cortical bone yield stress, before femur fracture was confirmed [11]. Therefore, it may be feasible to predict intraoperative fracture during insertion by performing finite element analysis in conjunction with sound frequency measurement.

We consider the difference in the patterns of hammering sound frequency exhibited by the two stems to be due to differences in the stem designs. Although both stems have a rectangular cross-section, the modified CLS stem has three fins, whereas the SL-PLUS stem has five holes. The pattern of the peak frequency of hammering sound may also vary with stem design.

Several limitations in the present study must be discussed. The artificial femurs used may have affected the susceptibility of the femurs to fracture, i.e., the artificial femurs may have increased the risk of fracture; this may have been the reason for fracture by a smaller number of hammer impacts in previous cases $[13,14]$. 


\section{CONCLUSION}

We found that the peak frequency produced by hammering during stem insertion decreased immediately after the first impact and before periprosthetic fracture, and that the pattern of frequency change differed with the artificial model of the stem insert. These findings may be useful for assessing fixation and predicting fracture risk in clinical settings.

\section{CONFLICTS OF INTEREST}

The authors declare no conflicts of interest regarding the publication of this paper.

\section{REFERENCES}

1. Byrick, R.J. (1997) Cement Implantation Syndrome: A Time Limited Embolic Phenomenon. Canadian Journal of Anesthesia, 44, 107-111. https://doi.org/10.1007/BF03012996

2. Moroni, A., Faldini, C., Piras, F. and Giannini, S. (2000) Risk Factors for Intraoperative Femoral Fractures during Total Hip Replacement. Annales Chirurgiae et Gynaecologiae, 89, 113-118.

https://www.ncbi.nlm.nih.gov/pubmed/10905677

3. Schwartz, J.T., Mayer, J.G. and Engh, C.A. (1989) Femoral Fracture during Non-Cemented Total Hip Arthroplasty. Journal of Bone and Joint Surgery: American Volume, 71, 1135-1142.

https://doi.org/10.2106/00004623-198971080-00003

4. Götze, C., Steens, W., Vieth, V., Poremba, C., Claes, L. and Steinbeck, J. (2002) Primary Stability in Cementless Femoral Stems: Custom-Made versus Conventional Femoral Prosthesis. Clinical Biomechanics, 17, 267-273. https://doi.org/10.1016/S0268-0033(02)00012-8

5. Lindahl, H. (2007) Epidemiology of Periprosthetic Femur Fracture around a Total Hip Arthroplasty. Injury, 38, 651-654. https://doi.org/10.1016/j.injury.2007.02.048

6. Pastrav, L., Jaecques, S., Jonkers, I., Perre, G. and Mulier, M. (2009) In Vivo Evaluation of a Vibration Analysis Technique for the Per-Operative Monitoring of the Fixation of Hip Prostheses. Journal of Orthopaedic Surgery and Research, 4, 10. https://doi.org/10.1186/1749-799X-4-10

7. Sakai, R., Kikuchi, A., Morita, T., Takahira, N., Uchiyama, K., Yamamoto, T., Moriya, M., Uchida, K., Fukusima, K., Tanaka, K., Takaso, M., Itoman, M. and Mabuchi, K. (2011) Hammering Sound Frequency Analysis and Prevention of Intraoperative Periprosthetic Fractures during Total Hip Arthroplasty. HIP International, 21, 718-723. https://doi.org/10.5301/HIP.2011.8823

8. Sakai, R., Takahashi, A., Takahira, N., Uchiyama, K., Yamamoto, T., Uchida, K., Fukusima, K., Moriya, M., Takaso, M., Itoman, M. and Mabuchi, K. (2011) Hammering Force during Cementless Total Hip Arthroplasty and Risk of Microfracture. HIP International, 21, 330-335. https://doi.org/10.5301/hip.2011.8408

9. Taylor, W.R., Roland, E., Ploeg, H., Hertig, D., Klabunde, R., Warner, M.D., Hobatho, M.C., Rakotomanana, L. and Clift, S.E. (2002) Determination of Orthotropic Bone Elastic Constants Using FEA and Modal Analysis. Journal of Biomechanics, 35, 767-773. https://doi.org/10.1016/S0021-9290(02)00022-2

10. Sakai, R., Itoman, M. and Mabuchi, K. (2006) Assessments of Different Kinds of Stems by Experiments and FEM Analysis: Appropriate Stress Distribution on a Hip Prosthesis. Clinical Biomechanics, 21, 826-833. https://doi.org/10.1016/j.clinbiomech.2006.03.008

11. Evans, F.G. (1969) The Mechanical Properties of Bone. Artificial Limbs, 13, 37-48.

http://www.oandplibrary.net/al/1969_01_037.asp

12. Schneider, E., Kinast, C., Eulenberger, J., Wyder, D., Eskilsson, G. and Perren, S.M. (1989) A Comparative Study of the Initial Stability of Cementless Hip Prosthesis. Clinical Orthopaedics and Related Research, 248, 200-209. https://www.ncbi.nlm.nih.gov/pubmed/2805480 
https://doi.org/10.1097/00003086-198911000-00032

13. Rosenstein, A.D., Bulstrode, C.J., Smith, M., Cunningham, J.L. and Turner-Smith, A.R. (1989) The Differentiation of Loose and Secure Femoral Implants in Total Hip Replacement Using a Vibrational Technique: An Anatomical and Pilot Clinical Study. Proceedings of the Institution of Mechanical Engineers, Part H: Journal of Engineering in Medicine, 203, 77-81. https://doi.org/10.1243/PIME_PROC_1989_203_014_01

14. Jaecques, S.V., Pastrav, C., Zahariuc, A. and Van der Perre, G. (2004) Analysis of the Fixation Quality of Cementless Hip Prostheses Using a Vibrational Technique. ASME 7 th Biennial Conference on Engineering Systems Design and Analysis, 2, 443-456. https://www.researchgate.net/publication/239581322

https://doi.org/10.1115/ESDA2004-58581 